\title{
ELEVEAN YEAR VARIATION IN HIGHER HARMONICS IN DAILY VARIATION OF COSMIC RAY INTENSITY ON QUIET DAYS AT EQUATORIAL AND HIGH LATITUDE NEUTRON MONITORING STATIONS
}

\author{
M. K. Richharia \\ Department of Physics \\ Govt. Model Science College, Jabalpur (M.P.) India
}

\begin{abstract}
The purpose of this investigation is establish eleven year variation in cosmic ray intensity particularly in third harmonic after using cosmic ray intensity neutron monitor data on sixty quiet days. It has been observed that in spite of abrupt variation in amplitude and phase of third harmonic of cosmic ray intensity, the amplitude of third harmonic is relatively larger during the declining phase of solar cycle twenty one as it is observed during the declining phase of earlier solar cycle twenty. Therefore, this enhancement establishes the eleven year variation in third harmonic of cosmic ray intensity on quiet days at equatorial neutron monitoring stations. Further, no systemic change have been noticed in the amplitude and phase of fourth harmonics of cosmic ray intensity on quiet days, but the amplitude of fourth harmonic during the year 1981 and 1991 having the same value at equatorial neutron monitoring stations.
\end{abstract}

\section{INTRODUCTION:}

The continuous outward flow of solar wind and frozen-in magnetic field produces time variations in cosmic ray intensity of different periodicities, viz. 22 years, 11 years, 27 days and 24 hours. The systematic study of the time variations of relativistic cosmic rays started some 60 years ago by using the ground based detectors. The ground based observations, its anisotropies and their relationship with other 
geomagnetic and interplanetary parameters, provide the base to understand the time variation characteristics of cosmic ray intensity.

Daily variation (First, Second, Third and Fourth harmonics) in cosmic ray intensities arise from anisotropies produced in the interplanetary medium. Daily variations are recorded by ground based detectors once a day as their asymptotic cones of acceptance sweep through the directions containing the spatial anisotropy. Detailed studies in this field were reviewed in the past by several researchers. The higher harmonics of cosmic ray daily variation such as semi-diurnal and tri-diurnal variation were interpreted on the basis of pitch angle distribution theory. During the past decades, the characteristics of tridiurnal variation (third harmonic of the daily variation) have been studied by a number of researchers. They have mostly used the data from the worldwide network of high counting rate neutron detectors [18].

\section{ANALYSIS OF THE DATA}

Solar daily variation has been studied in terms of helio-magnetic activity. A new concept of data analysis has been introduced for studying the long / short term daily variation in CR intensity recorded with neutron monitors. Fourier technique has been applied on different types of group of days chosen according to their different geomagnetic condition.

1. All days : this means al the $365 / 366$ days in year. Thus, these days are termed as $A D$. of course ignoring the days with abrupt charges.

2. Quiet days : Those days on which the transient magnetic variation are regular and smooth are said to be magnetically quiet or $Q$ days. The criteria is based upon Ap and Kp values. There are two types of days. 
i) 60 Quiet days : According to solar geophysical data (SGD) lowest mean order number are the five quietest days in a month. Thus, $60 \mathrm{Q}$ days in year; termed as $60 \mathrm{QD}$.

ii) 120 Quiet days : First ten quiet days in a month. Thus, 120 $Q$ days in a year; termed as 120 Quiet days.

The pressure corrected hourly CR intensity data (corrected for meteorological effects) on geo magnetically five quietest days (QD) in every month for Tokyo (Lat: $35.75^{\circ} \mathrm{N}$; Cutoff rigidity; $11.61 \mathrm{GV}$; Longitude: $139.72^{0} \mathrm{E}$; Altitude: $20 \mathrm{~m}$ ), Mount Nourikura (Lat: $36.12^{0} \mathrm{~N}$; Cutoff rigidity: $11.39 \mathrm{GV}$; Longitude: $137.56^{\circ} \mathrm{E}$; Altitude: $27.7 \mathrm{~m}$ ) and Inuvik (Lat : $68.35^{\circ} \mathrm{N}$; Cutoff rigidity: $0.18 \mathrm{GV}$; Longitude: $226.27^{0} \mathrm{E}$; Altitude: $21 \mathrm{~m}$ ) neutron monitoring stations for the period 1978-94, have been used in Fourier analysis. After applying ther trend corrections, such a set of data have been subjected to Harmonic analysis for each day [9]. The average values of the amplitude and phase (local time of the station) of the third (tri diurnal) harmonics and Fourth harmonics (quart-diurnal) on yearly basis have been obtained. According to solar geophysical data five quietest days are selected in a month; thus 60 quietest days are obtained in a year. These days are called international quiet days (QD). The days with extra ordinary large amplitude, if any, have not been considered. Further, the variation in the tri-diumal anisotropy with the reversal of polarity of solar magnetic field (PSMF) on 60 QD has been also investigated. Also all those days are discarded having more than three continuous hourly data missing.

\section{RESULT AND DISCUSSION}

The yearly average amplitude and the phase of the third harmonics of daily variation for Inuvik, during the period 1978-94 and Tokyo Mount Nourikura Neutron Monitoring Station during the period 1980-90 have been plotted in Fig. 1 on QD. It is quite apparent from Fig. 1 that there is no systematic change in the amplitude of third harmonics 
on QDs. Nevertheless, the amplitude of third harmonics on QD remain relatively larger during the declining phase of solar cycle 21 and 22 as compared with the declining phase of the earlier solar cycle 20 at equatorial stations. This enhancement explicitly point out 11 year periodicity at equatorial neutron monitoring station $[10,11]$. Further, the amplitude of the third harmonics on QD has low values during minimum solar activity period and the amplitude of third harmonics of daily variation in the year 1980 has quite abruptly increased at Mount Nourikura Neutron monitoring station. The likely one of the cause of such type of variation could be changing threshold cut off rigidity from $1.02 \mathrm{GV}$ to $1.15 \mathrm{GV}[12-14]$.

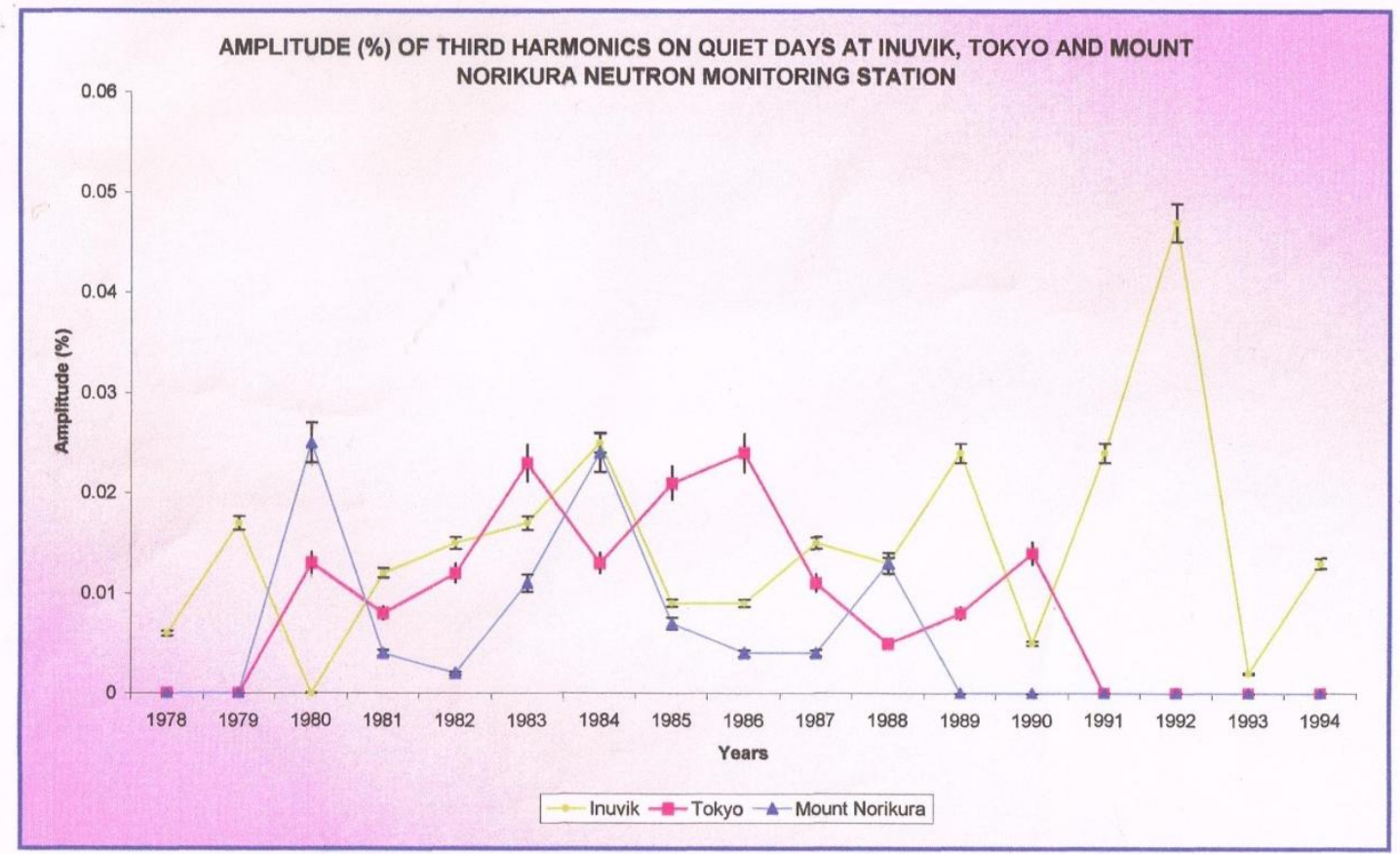

Figure 1

Furthermore, The amplitude of third harmonics of daily variation on QD is observed to be significantly low during the year 1981 as well as in 1990, which coincides with phase reversal of the solar poloidal magnetic filed and the amplitude of third harmonics on QD is low during 1985 and 1986, the year of minimum solar activity. at Inuvik neutron 
monitoring station The cause of such changes may be the increase of interplanetary magnetic field irregularities (IMF). The amplitude of third harmonics of daily variation on quiet days during the year 1978 and 1990 (Maximum Solar activity period of solar cycle 21 and 22) having same values. A clear 11 year type variation is established at high latitude neutron monitoring station [15-18]. It is also observed from Fig. 2 that there is no systematic change in the phase of third harmonics of daily variation in cosmic ray intensity on QD. However, in the year 1990, the phase shifted to later hours, when the polarity of solar magnetic field in the northern hemisphere has changed from negative to positive. The polarity dependence of the phase shift change has been interpreted as a result due to the change of $C R$ density distribution in space caused by the difference of $\mathrm{CR}$ drift motion in the positive and negative polarity state.

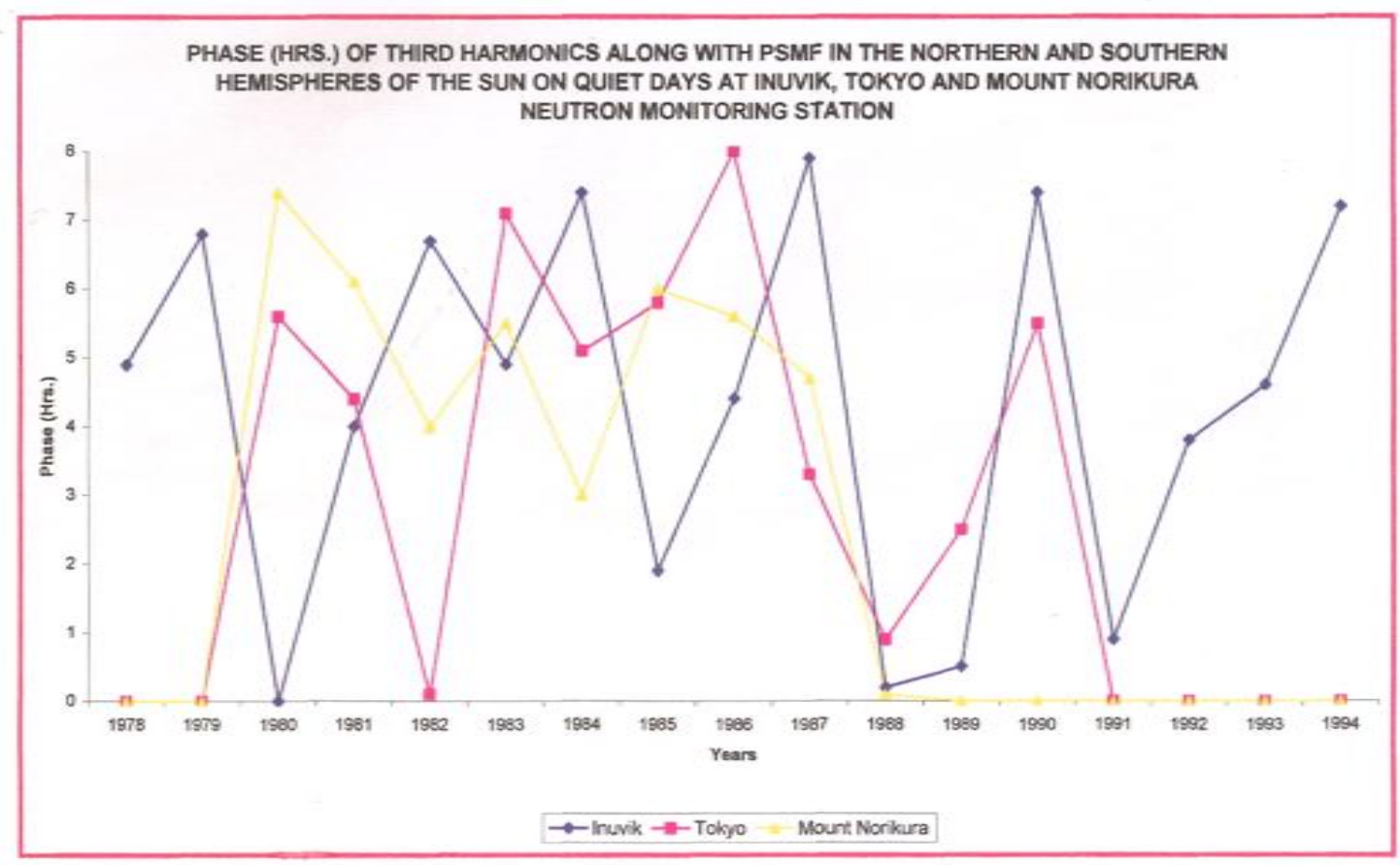

Figure 2

The annual average amplitude and phase (Hrs) (in local time of station) of the fourth harmonics of daily variation on QD's has been plotted in Fig. 3 and Fig. 4 during the year 1978-94 for Inuvik neutron monitoring station and during the year 1980-90 for Tokyo and Mount 
Nourikura neutron monitoring station. It is quite apparent from the Fig. 3 and Fig. 4 that there is no systematic change in the amplitude and phase of fourth harmonic of daily variation on QD. However the amplitude of fourth harmonics of daily variation on QD during the interval 1987-90 shown an increasing trend continuously in association with phase of fourth harmonics shifted to earlier hours at Tokyo Neutron monitoring station. The amplitude of fourth harmonics on QD has larger value during the declining phase of solar cycle 21 i.e., 1984-86 at Mount Nourikura neutron monitoring station. Thus enhancement explicitly point 11 year periodicity in fourth harmonic of daily variation on QD [17]. The remarkable change has been noticed in the phase continuously shift to early hours from 1980 to 1983 and then shifted to later hours during from 1983 to 1987 at Mount Nourikura Neutron monitoring station. Further, no significant change have been noticed in amplitude and phase of during minimum solar activity period 1983-86 of solar cycle 21 at both the neutron monitoring station.

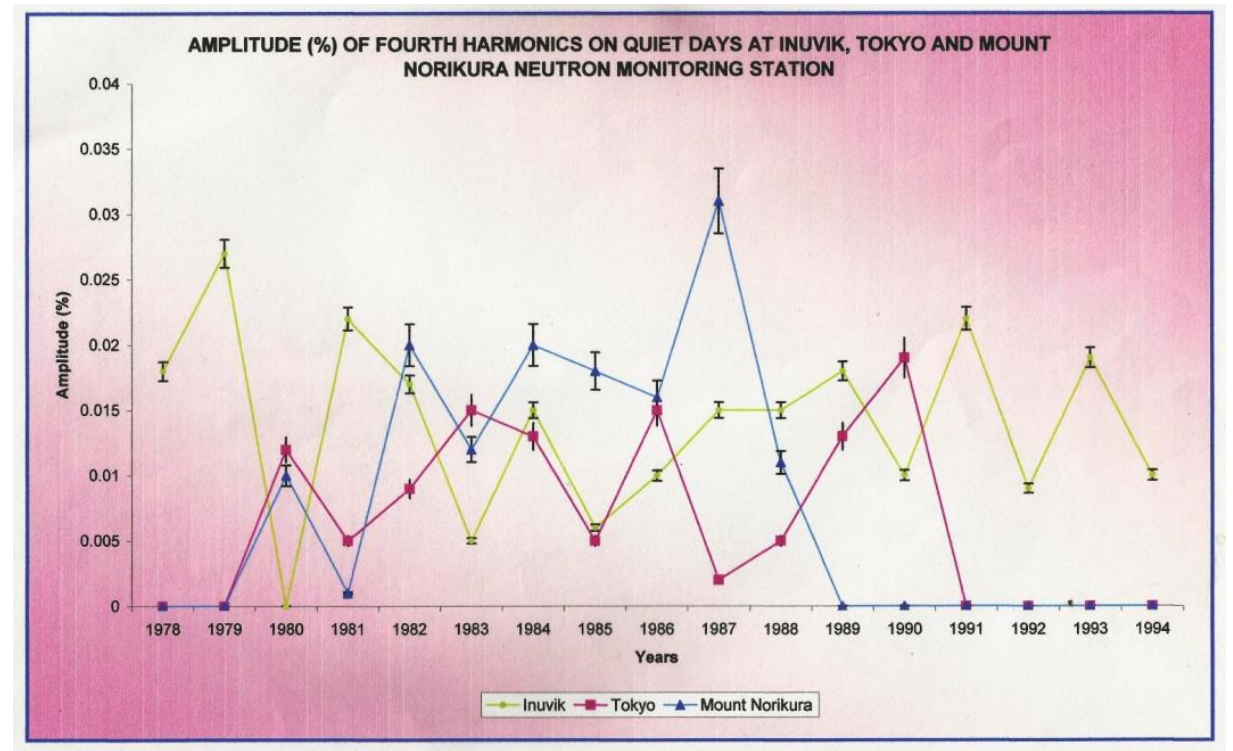

Figure 3 


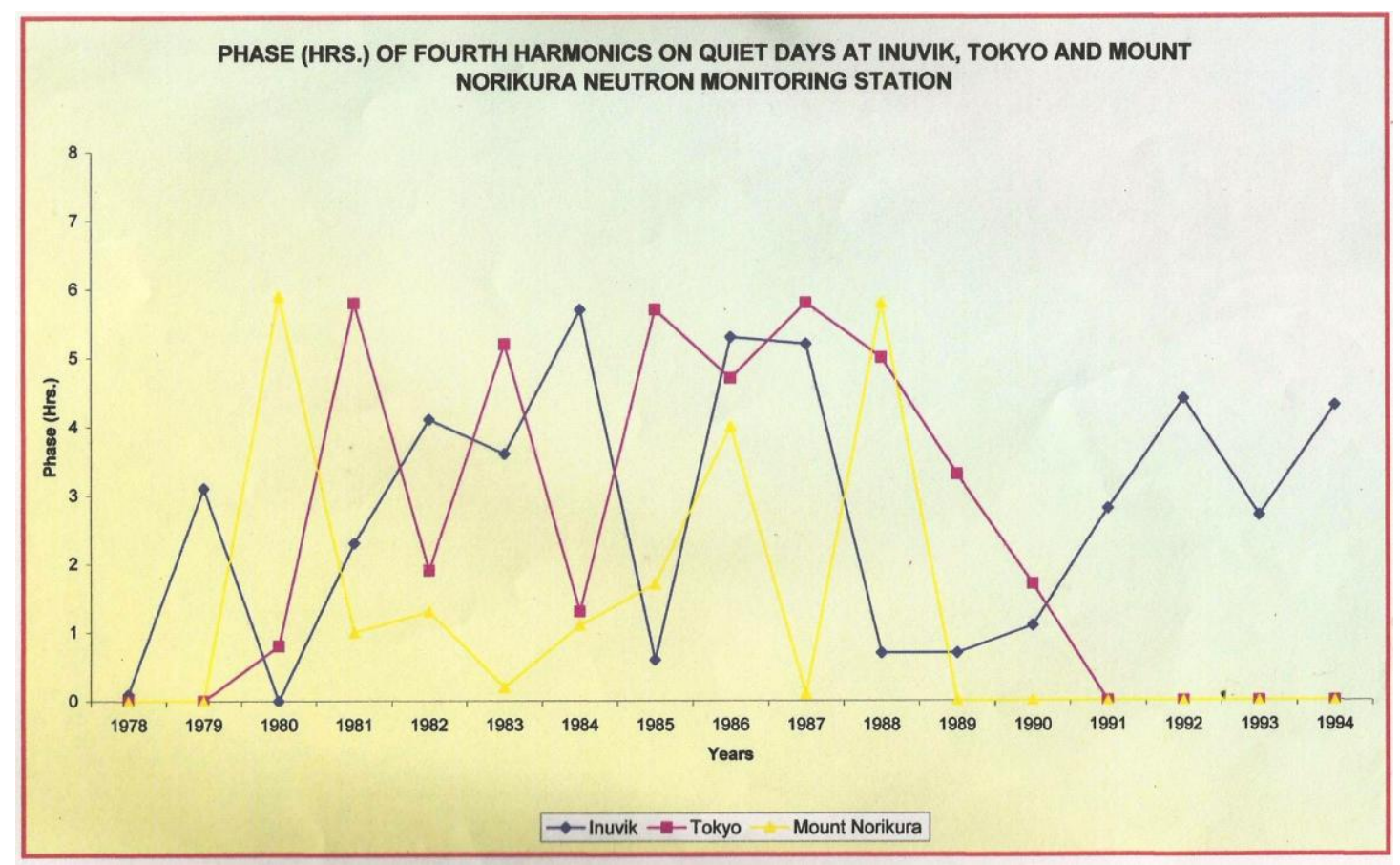

Figure 4

\section{ACKNOWLEDGEMENT}

The author are indebtend to various experimental groups; in particular, Prof. M. Bercovitch, K. Nagashima and Miss Aoi Inoue for providing neutron monitor data.

\section{REFERENCES :}

1. D. Venkatesan and Badruddin, Space Science Rev., 52, 121 (1990) and reference there in.

2. Fujii, A., Nagashima, K., Fujimoto, K., Ueno, H. And Kondo, I. 1971. $12^{\text {th }}$ ICRC, Hobart Tasmania, 2, 666.

3. Ahluwalia, H.S. and Singh, S. 1973 a, Proc. $13^{\text {th }}$ Int. Cosmic Ray Conf., Australia, 2:948.

4. Ahluwalia, H.S. and Singh, S. 1973 b, Proc. $13^{\text {th }}$ Int. Cosmic Ray Conf. Australia, 5:3129.

5. Pomerantz, M.A. and Duggal, S.P. 1971,Spacee Sci. Rve., 12.75.

6. Rao, U.R. 1972, Space Sci., Rev., 12, 719.

7. Agrawal, S.P. 1981, Journal Geophys, Res. 86: 10115. 
8. Kamlesh Singh and Pankaj K. Shrivastava, 2010, Indian Journal of Radio and Space Physics, 341.

9. Kumar, S. Agrawal, R., Mishra, R. And Dubey, S.K. 2002, Bull Astronomical Soc. India, 30, 451.

10. El Borie, M.A.,Sabba, I.S. Darwish, A. A. and Bishra, A.A. 1995a, $24^{\text {th }}$ Int. Cosmic Ray Conf., Rome, Italy, 4:619

11. Richharia, M. K. 2011, $32^{\text {nd }}$ International Cosmic Ray Conference, Beijing China, 11, S.H. 3: 4, 127.

12. Shea, M. A. and Smart, D. F. 1983, $18^{\text {th }}$ Int. Cosmic Ray Conf. Bangalore, 3:411.

13. Shea, M. A. and Smart, D. F. $200127^{\text {th }}$ Int. Cosmic Ray Conf. Hemberg, 3:4063.

14. Smart, D. F. and Shea, M. A. 1987. $20^{\text {th }}$ Int. Cosmic Ray Conf. Moscow, 4:204.

15. Kumar, S., Richharia, M.K. and Shrivastava, S.K.,1999. Proceeding National Academy of Science, India, 69(A), II.

16. Shea, M. A. and Smart, D. F. $200127^{\text {th }}$ Int. Cosmic Ray Conf. Hemberg, 3:4063.

17. El Borie, M.A.,Sabba, I.S. Darwish, A. A. and Bishra, A.A. 1995a, $24^{\text {th }}$ Int. Cosmic Ray Conf., Rome, Italy, 4:127.

18. Richharia, M.K. Proceeding of Sciences, $34^{\text {th }}$ Int. Cosmic Ray Conf. 2015, 160. 